Bioscientia Medicina: Journal of Biomedicine \& Translational Research

Journal Homepage: www.bioscmed.com

\title{
Dermatoglyphy in Breast Cancer Patients: A Systematic Review
}

\section{Rara Inggarsih ${ }^{*}$, Akhyar Dyni Zakyah ${ }^{2}$, Lusia Hayati ${ }^{1}$, Joko Marwoto ${ }^{1}$, Septi Purnamasari ${ }^{1}$, Arwan Bin Laeto ${ }^{3}$, Masayu Farah Diba ${ }^{4}$,}

${ }^{1}$ Department of Biology, Faculty of Medicine, Universitas Sriwijaya, Palembang, Indonesia

${ }^{2}$ Department of Dentistry, Faculty of Medicine, Universitas Sriwijaya, Palembang, Indonesia

${ }^{3}$ Department of Physiology, Faculty of Medicine, Universitas Sriwijaya, Palembang, Indonesia

${ }^{4}$ Department of Microbiology, Faculty of Medicine, Universitas Sriwijaya, Palembang, Indonesia

\section{A R T I C L E I N F O \\ Breast neoplasms \\ Pregnancy \\ Human BRCA2 protein \\ Mutation \\ Estrogen}

\section{*Corresponding author: \\ Rara Inggarsih \\ E-mail address: rarainggarsih@fk.unsri.ac.id}

All authors have reviewed and approved the final version of the manuscript.

https://doi.org/10.32539/bsm.v5i11.368

\begin{abstract}
A B S T R A C T
Background. Breast cancer is one of the four types of cancer among women and is the most frequently diagnosed in most countries. Breast cancer occurs due to DNA damage and genetic mutations affected by exposure to estrogen, inheritance of damaged DNA, or pro-cancer genes such as BRCA1 and BRCA2. Therefore, a family history of ovarian cancer or breast cancer increases the risk of developing breast cancer. The embryo of the breast develops around the age of 6 weeks of pregnancy. Similar to breast development, fingerprint patterns also develop during the 6-13 weeks of pregnancy. Thus, the genetic message contained in the genome occurred during that period and was reflected in the dermatoglyphic pattern. Methods. The literature search was systematically used using PubMed, Cochran, Google scholar, and other Gray literature between 2010-2020. Of the 69 publications identified, 21 met the criteria and were included in the review. The review is carried out following the provisions of PRISMA (Preferred Reporting Items for Systematic Review). Results. This systematic review showed fairly consistent findings in breast cancer patients who tended to have more whorl fingerprint patterns and larger ATD angles. For radial loops, ulnar loops and arches were minor compared to the control group potential as an initial screening tool in at-risk groups. Conclusion. Long-term and follow-up studies with larger sample sizes in various ethnicities are needed to validate dermatoglyphics in anthropometric measurements as a promising marker of breast cancer.
\end{abstract}

\section{Introduction}

There were approximately 2.1 million new cases of breast cancer diagnosed worldwide in 2018, which is 1 in 4 cancer cases among women. This disease is the most commonly diagnosed cancer in most countries (154 out of 185 countries in the world) and also the leading cause of cancer death in more than 100 countries. ${ }^{1}$ There is still limited knowledge about how geographic variation is related to certain etiological factors. Breast cancer incidence rates have increased in most transition countries over the past few decades. Some of the highest increases occur in historically relatively low breast cancer rates, such as South America, Africa, and Asia. ${ }^{2}$

Breast cancer occurs due to DNA damage and genetic mutations that can be affected by exposure to estrogen. Sometimes it is due to inherited defective DNA or pro-cancer genes such as BRCA1 and BRCA2. Therefore, a family history of ovarian cancer or breast cancer increases the risk of developing breast cancer. In normal individuals, the immune system can attack cells with abnormal DNA or abnormal growth. However, 
this is not the case in breast cancer patients, leading to tumor growth and spread. ${ }^{3}$

The embryonic breast develops around six weeks of gestation as a solid growth of the epidermis in the underlying mesenchyme. ${ }^{4}$ Similar to breast development, fingerprint patterns also develop during 6-13 weeks of gestation. 5 Thus, the genetic message contained in the genome occurred during that period and was reflected in the dermatoglyphic pattern. Once established, the dermatoglyphic pattern does not change throughout life except in the event of a traumatic disturbance. ${ }^{6}$ Dermatoglyphics can be applied to study the genetic basis of breast cancer and can be used as a non-invasive, inexpensive, and effective screening tool in high-risk populations. ${ }^{7}$ Dermatoglyphics is the scientific study of fingerprint patterns, palms, hands, soles of humans and animals' feet, and toes. Genetically, the pattern formed is determined by the interaction of several genes to form a characteristic that distinguishes one from another. There are four general types of fingerprint patterns classified as whorl, ulnar loop, radial loop, and arch (figure 1). ${ }^{8}$

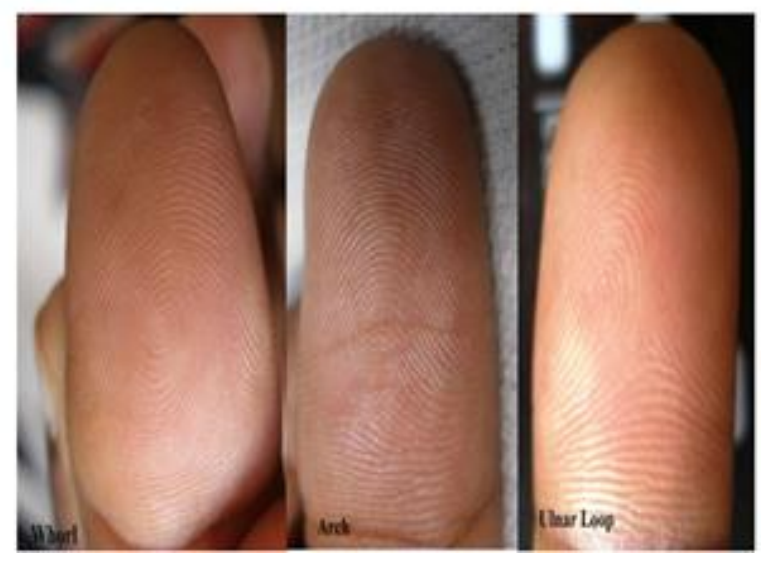

Figure 1. fingerprint pattern variation

In addition to qualitative parameters such as fingerprint patterns, quantitative parameters are usually investigated, including total finger ridge count (TFRC), absolute finger ridge count (AFRC), a-b ridge count, and ATD Angle. TFRC is the number of segments or strokes of the ten distal phalanxes. The calculation method is done by making a line drawn from the triradius point to the core but excluding the radius and core points. For a whorl pattern that has two triradius, the most sides are counted. A loop pattern that only has one triradius means that only one side will be counted the stroke. Then because the arch pattern does not have a triradius, the number of strokes is not counted.9 The absolute finger ridge count (AFRC) describes the number of strokes of all fingers, including two strokes of the whorl pattern (figure 2). ${ }^{10}$



Figure 2. Counting the ridge count on each finger

a-b Ridge Count is done by drawing a straight line connecting the digital triradii ' $a$ ' and ' $b$ ' and then calculating the entire segment or stroke between them. ${ }^{11}$ The magnitude of the Axial Triradius Digital 
(ATD) angle can be calculated by determining the location of each triradius starting from the tip of the second finger to the tip of the fifth finger. Point a is the triradius point on the second finger, point $b$ on the third finger, point $\mathrm{c}$ on the IV finger, and point $\mathrm{d}$ on the
$\mathrm{V}$ finger. The point $(\mathrm{t})$ is the point in the middle of the base of the palm.12 The purpose of this study was to systematically assess and evaluate the dermatoglyphic parameters that are associated and dominant found in breast cancer patients.

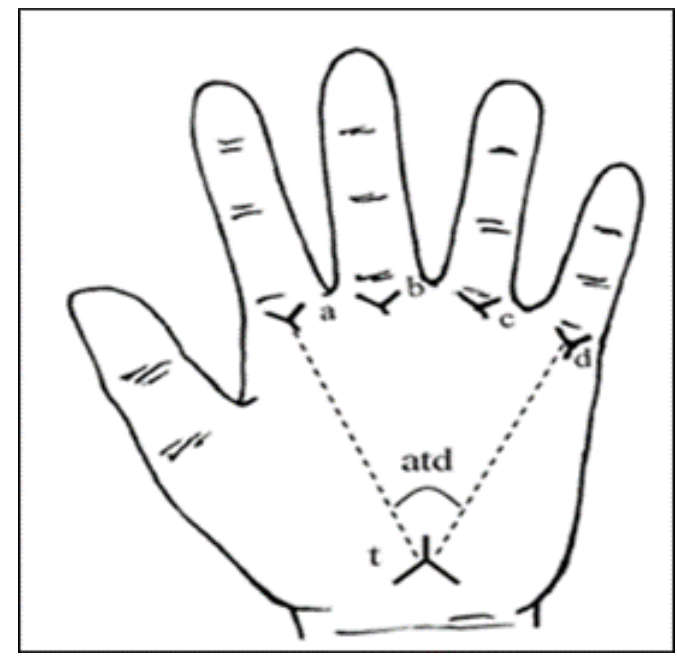

Figure 3. a-b ridge count and ATD angle

\section{Methods}

The method used in this study is a systematic review to collect, identify, evaluate and interpret the dermatoglyphic parameters that are associated and dominant found in breast cancer patients. The search for articles or literature was obtained using the PubMed, Cochrane, ProQuest search engine facilities, while Gray Literature was obtained using Google Scholar, WorldCat, and Science Direct with a publication time limit of 2010-2020.

The general search strategy used for the PubMed database consists of MESH terms using Boolean Operators. The keywords used included "breast neoplasms", "breast cancer", "breast tumors", "breast carcinoma", "mammary cancer", "ductal carcinoma", "dermatoglyphics", "dermatoglyphic", "plantar prints", " palmar pattern", "dermatoglyphical". The term "breast cancer and dermatoglyphics" is used in searches in the Cochrane, Google Scholar, and other Gray Literature databases as well as article searches using the Retrospective Snow Balling method.

The research obtained was identified and screened based on predetermined criteria. Inclusion criteria were case-control, cohort, cross-sectional studies that evaluated the relationship between dermatoglyphic parameters, both qualitative assessment (fingerprint pattern) and quantitative assessment (TFRC, AFRC, Triradial RC, ab ridge count, $<$ ATD, $<$ ADT, $<$ DAT) with breast cancer or dermatoglyphic differences between case and control groups; dermatoglyphics of the hands; research using English or Bahasa. Exclusion criteria in the form of descriptive research, case reports, case studies, and reviews; the control group had a personal or family history of cancer or other genetic diseases. The systematic review of the research followed the method according to the provisions of PRISMA (Preferred Reporting Items for Systematic Review). Data analysis begins by following the steps of the PRISMA method, including identification, screening, and feasibility of the articles to be analyzed. Articles that meet the criteria will be reviewed to list the dermatoglyphic parameters associated and dominantly found in breast cancer patients. The findings will be recorded and presented in a table accompanied by explanations, discussions, and drawing conclusions. 


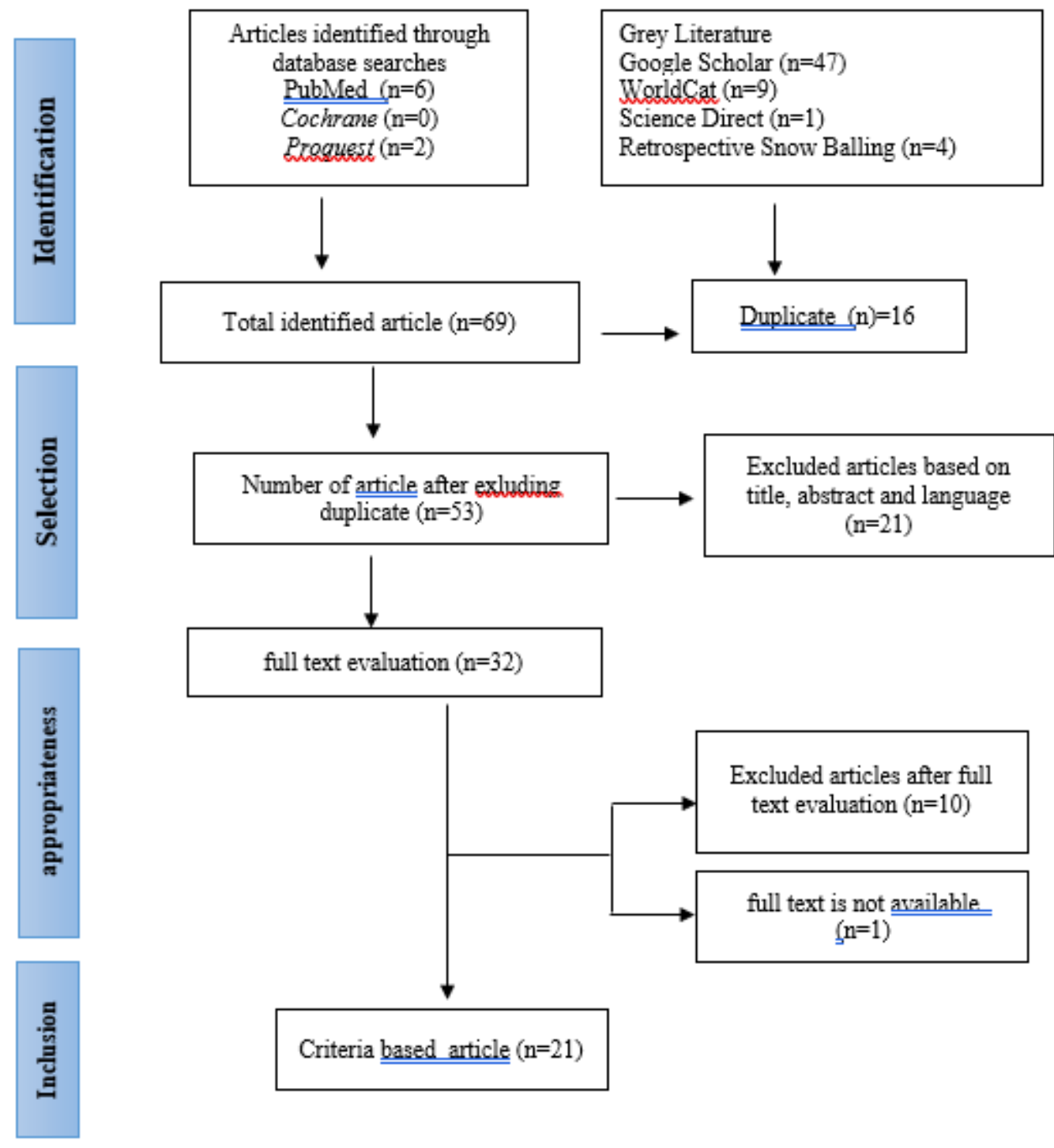

Figure 4. Flowchart of systematic review research methods using PRISMA.

\section{Result}

A search of the scientific database identified 69 publications using predefined keywords (figure 4). Of these, 48 studies were not suitable for further analysis because it is a duplicate $(\mathrm{n}=16)$, the title filter is not suitable for this study ( $\mathrm{n}=5$ ), excluded because of the type of research ( $\mathrm{n}=9)$, there is no association of dermatoglyphics and breast cancer or significant differences between case and control groups $(n=5)$, examined other parameters such as mainline pattern, fluctuating asymmetry, thenar, hypothenar, DNA polymorphism $(n=5)$, dermatoglyphic observations only on toes $(n=1)$, the control group was not stated whether they had a personal or family history of cancer or other genetic diseases $(n=3)$, articles in Chinese $(n=1)$, articles not published in journals $(n=1)$, criteria which are used to explain the research results is different from the average of other studies and cannot be converted $(n=1)$, full-text articles cannot be accessed $(\mathrm{n}=1)$.

A total of 21 articles ${ }^{13-33}$ met the criteria for review in this study. Of the 21 studies, 15 were conducted in India $13,14,16-18,20-22,25-29,32,33,2$ in Bulgaria 15,19, 3 in Bosnia-Herzegovina 23,24,30, and 1 in Egypt ${ }^{31}$. Several studies were included in the study and according to the selected criteria examined qualitative data on the relationship between breast cancer and whorl patterns as many as 14 studies 4,16,18,20,21-23,25-28,30-32. Breast cancer and radial loop pattern were 9 studies 14,16,21$23,25,26,31,32$. The following relates to the ulnar loop 
pattern as many as 9 studies 14,16,21-23,25,26,30-32. Furthermore, the association between breast cancer and arch patterns was 14 studies 14,16,18,20,21-23,25-28,3032. Breast cancer and the combination of ulnar and radial loop patterns were 5 studies 18,20,27,28,30. Several studies separated the right and left hand categories and the number of patterns $<6$ or $>6$.

The quantitative data studied included the relationship between breast cancer and TFRC in 9 studies 15,18,25,27-30,32,33. One study discussed the relationship between breast cancer and AFRC18. Furthermore, the relationship between breast cancer and a-bridge count was discussed by 8 studies $^{2,14,18,19,25,29,32,33}$. 10 studies reported the relationship between breast cancer and ATD angle $2,14,17,24,25,27,29,30,32,33$ while the ADT angle and breast cancer were discussed by 2 studies 27,32. There is a relationship between breast cancer and DAT angle in 1 study ${ }^{27}$. A total of 2 studies evaluated the association between breast and triradial cancer ${ }^{14,25}$, Dankmeijer index Furuhata index 20 , and total pattern intensity 29 . Similar to qualitative data, many studies separate the right and left-hand categories and several variations of other categories.

Table 1. breast cancer and whorl pattern

\begin{tabular}{|c|c|c|c|c|c|c|c|}
\hline \multicolumn{8}{|c|}{$\begin{array}{l}\text { Subgroup criteria } \\
\text { P: female, aged } 25-60 \text { years old } \\
\text { I: breast cancer based on histopathological evaluation } \\
\text { C: non- breast cancer } \\
\text { O: dermatoglyphy }\end{array}$} \\
\hline No & Results & $\begin{array}{l}\text { Number of } \\
\text { studies }\end{array}$ & Participants & $\begin{array}{l}\text { P-value/ } \\
\text { ratio/ } \\
\text { ratio/ } \\
\text { confidence } \\
\text { interval }\end{array}$ & $\begin{array}{l}\text { risk } \\
\text { odd }\end{array}$ & $\begin{array}{l}\text { Individual } \\
\text { bias risk }\end{array}$ & Notes \\
\hline 1 & $\begin{array}{l}\text { Whorl pattern } \\
\text { percentage in cases > } \\
\text { control }(42,80>23,80)\end{array}$ & $\begin{array}{l}1 \\
2012)\end{array}$ & 100 & $\mathrm{p}<0,05$ & & $51,04 \%$ & \multirow{11}{*}{$\begin{array}{l}\text { Of the } 20 \\
\text { studies, there } \\
\text { were } 5 \text { studies } \\
\text { with p value } \\
<0.05 \text { and a good } \\
\text { bias assessment } \\
>\quad 50 \% \text { which } \\
\text { stated that the } \\
\text { whorl pattern } \\
\text { was more } \\
\text { common in } \\
\text { breast cancer } \\
\text { patients than the } \\
\text { control group. }\end{array}$} \\
\hline 2 & $\begin{array}{lr}\text { Whorl } & \text { pattern } \\
\text { percentage } & \text { in } \\
\text { cases }>\text { control } & \\
(39,70>27,00) & \\
\end{array}$ & $\begin{array}{l}1 \text { (Paranjape, } \\
2015) \text { ) }\end{array}$ & 200 & $\mathrm{p}<0,001$ & & $52,60 \%$ & \\
\hline 3 & $\begin{array}{l}\text { Persentase pola whorl } \\
\text { pada kasus }>\text { kontrol } \\
(81,00>80,00)\end{array}$ & $\begin{array}{l}1 \text { (Krishnan, } \\
2016)\end{array}$ & 200 & $\mathrm{p}>0,05$ & & $45,83 \%$ & \\
\hline 4 & $\begin{array}{l}\text { Total value whorl } \\
\text { pattern in cases }>\text { control } \\
(466,00>374,00)\end{array}$ & $\begin{array}{l}1 \\
2016)\end{array}$ & 200 & $\mathrm{p}<0,05$ & & $60,42 \%$ & \\
\hline 5 & $\begin{array}{l}\text { Total value whorl } \\
\text { pattern in cases }>\text { control } \\
(338,00>290,00)\end{array}$ & $\begin{array}{l}1 \text { (Meghala } \\
2020)\end{array}$ & 200 & $\mathrm{P}=0,0001$ & & $42,71 \%$ & \\
\hline 6 & $\begin{array}{l}\text { Total value whorl } \\
\text { pattern in cases }<\text { control } \\
(358,00<737,00)\end{array}$ & $\begin{array}{l}1 \\
2020)\end{array}$ (Singh, & 290 & $p<0,001$ & & $48,96 \%$ & \\
\hline 7 & $\begin{array}{l}\text { Whorl pattern }<6 \text { on } \\
\text { cases }<\text { control } \\
(43,00>72,00)\end{array}$ & $\begin{array}{l}1 \text { (Musanovic, } \\
2019)\end{array}$ & 100 & $p>0,05$ & & $52,60 \%$ & \\
\hline 8 & $\begin{array}{l}\text { Total value of } \begin{array}{r}\text { whorl } \\
\text { pattern }\end{array} \\
\text { cases }>\text { control } \\
(50,00>39,00)\end{array}$ & $\begin{array}{l}1 \\
2012)\end{array}$ & 100 & $\mathrm{p}<0,05$ & & $47,92 \%$ & \\
\hline 9 & $\begin{array}{l}\text { Total value whorl } \\
\text { pattern right hand on } \\
\text { cases }<\text { control }(106,00< \\
139,00)\end{array}$ & $\begin{array}{l}1 \text { (Raizada, } \\
2013)\end{array}$ & 200 & $\mathrm{p}<0,05$ & & $56,77 \%$ & \\
\hline 10 & $\begin{array}{l}\text { Total value whorl } \\
\text { pattern left hand on } \\
\text { cases }<\text { control }(101,00< \\
154,00)\end{array}$ & $\begin{array}{l}1 \text { (Raizada, } \\
2013)\end{array}$ & 200 & $\mathrm{p}<0,001$ & & $56,77 \%$ & \\
\hline 11 & Total value whorl & (Sridevi, & 200 & $\mathrm{p}=0,076$ & & $55,73 \%$ & \\
\hline
\end{tabular}




\begin{tabular}{|c|c|c|c|c|c|}
\hline & $\begin{array}{l}\text { pattern } \\
\text { cases }<\text { control }(303,00< \\
340,00)\end{array}$ & 2010) & & & \\
\hline 12 & $\begin{array}{lr}\text { Whorl } & \text { pattern } \\
\text { percentage } & \text { on } \\
\text { cases }>\text { control } & (44,77> \\
38,89) & \end{array}$ & $\begin{array}{l}1 \\
\text { (Shrivastava, } \\
\text { 2019) }\end{array}$ & 218 & $\mathrm{p}=0,005$ & $43,23 \%$ \\
\hline 13 & $\begin{array}{l}\text { Whorl pattern }<6 \text { on } \\
\text { cases }>\text { control }(113,00 \\
>78,00\end{array}$ & $\begin{array}{l}1 \text { (Musanovic, } \\
2018 \text { ) }\end{array}$ & 232 & $\mathrm{p}=0,070$ & $60,94 \%$ \\
\hline 14 & $\begin{array}{l}\text { Whorl pattern }>6 \text { on } \\
\text { case }<\text { control }(19,00< \\
23,00\end{array}$ & $\begin{array}{l}1 \text { (Musanovic, } \\
2018 \text { ) }\end{array}$ & 232 & $p=0,070$ & $60,94 \%$ \\
\hline 15 & $\begin{array}{l}\text { Total value of whorl } \\
\text { pattern right hand on } \\
\text { cases }>\text { control }(230,00 \\
>120,00)\end{array}$ & $\begin{array}{l}1 \\
\text { (Abdelhamid, } \\
2020 \text { ) }\end{array}$ & 1000 & $\mathrm{p}<0.00001$ & $54,17 \%$ \\
\hline 19 & $\begin{array}{l}\text { Percentage } \\
\text { pattern on cases }> \\
\text { control }(53,20>15,80)\end{array}$ & $\begin{array}{l}1 \text { (Madhavi, } \\
\text { 2013) }\end{array}$ & 200 & - & $45,83 \%$ \\
\hline 20 & $\begin{array}{l}\text { Percentage } \begin{array}{r}\text { whorl } \\
\text { pattern on cases }< \\
\text { control }(16,20<56,00)\end{array} \\
\end{array}$ & $\begin{array}{l}1 \quad \text { (Madhavi, } \\
2013)\end{array}$ & 200 & - & $45,83 \%$ \\
\hline
\end{tabular}

Table 2. Breast cancer and loop radial pattern

\begin{tabular}{|c|c|c|c|c|c|c|c|}
\hline \multicolumn{8}{|c|}{$\begin{array}{l}\text { Subgroup criteria } \\
\text { P: female, aged } 25-60 \text { years old } \\
\text { I: breast cancer based on histopathological evaluation } \\
\text { C: non- breast cancer } \\
\text { O: dermatoglyphy }\end{array}$} \\
\hline No & Results & $\begin{array}{l}\text { Number of } \\
\text { study }\end{array}$ & $\begin{array}{l}\text { Total } \\
\text { participants }\end{array}$ & $\begin{array}{l}\text { P-value/ } \\
\text { ratio/ } \\
\text { ratio/ } \\
\text { confidence } \\
\text { interval }\end{array}$ & $\begin{array}{l}\text { risk } \\
\text { odd }\end{array}$ & $\begin{array}{l}\text { Individual } \\
\text { bias risk }\end{array}$ & Notes \\
\hline 1 & $\begin{array}{l}\text { Percentage loop radial } \\
\text { pattern on cases < } \\
\text { control }(3,00<4,00)\end{array}$ & 1 (Fulari, 2012) & 100 & $\mathrm{p}>0,05$ & & $51,04 \%$ & \multirow{8}{*}{$\begin{array}{l}\text { There were } 6 \\
\text { studies which } \\
\text { stated that the } \\
\text { radial pattern } \\
\text { was less in the } \\
\text { case group than } \\
\text { the control group } \\
\text { with p value < } \\
0.05 \text { and a good } \\
\text { bias assessment } \\
>50 \% \text {. }\end{array}$} \\
\hline 2 & $\begin{array}{l}\text { Percentage loop radial } \\
\text { pattern on cases < } \\
\text { control }(3,20<5,10)\end{array}$ & $\begin{array}{l}1 \text { (Paranjape, } \\
2015)\end{array}$ & 200 & $\mathrm{p}<0,05$ & & $52,60 \%$ & \\
\hline 3 & $\begin{array}{l}\text { Total loop radial pattern } \\
\text { on cases > control } \\
(25,00>15,00)\end{array}$ & $\begin{array}{l}1 \text { (Meghala, } \\
\text { 2020) }\end{array}$ & 200 & $\mathrm{p}=0,0001$ & & $42,71 \%$ & \\
\hline 4 & $\begin{array}{l}\text { Total loop radial pattern } \\
\text { on cases > control } \\
(47,00>36,00)\end{array}$ & 1 (Singh, 2020) & 290 & $\mathrm{p}<0,001$ & & $48,96 \%$ & \\
\hline 5 & $\begin{array}{l}\text { Amount of loop radial } \\
\text { pattern }>6 \text { on cases }< \\
\text { control }(31,00<40,00)\end{array}$ & $\begin{array}{l}1 \text { (Musanovic, } \\
2019)\end{array}$ & 100 & $p<0,05$ & & $60,94 \%$ & \\
\hline 6 & $\begin{array}{l}\text { Amount of loop radial } \\
\text { pattern }>6 \text { on cases }> \\
\text { control }(19,00>10,00)\end{array}$ & $\begin{array}{l}1 \quad \text { (Musanovic, } \\
2019)\end{array}$ & 100 & $\mathrm{p}<0,05$ & & $60,94 \%$ & \\
\hline 7 & $\begin{array}{l}\text { Total loop radial pattern } \\
\text { on cases }<\text { control }(1,00 \\
<4,40)\end{array}$ & 1 (Sukre, 2012) & 100 & $\mathrm{p}<0,05$ & & $47,92 \%$ & \\
\hline 8 & $\begin{array}{l}\text { Total loop radial pattern } \\
\text { right hand on cases < } \\
\text { control }(189,00< \\
296,00)\end{array}$ & $\begin{array}{l}1 \\
2013)\end{array}$ & 200 & $\mathrm{p}<0,001$ & & $56,77 \%$ & \\
\hline
\end{tabular}




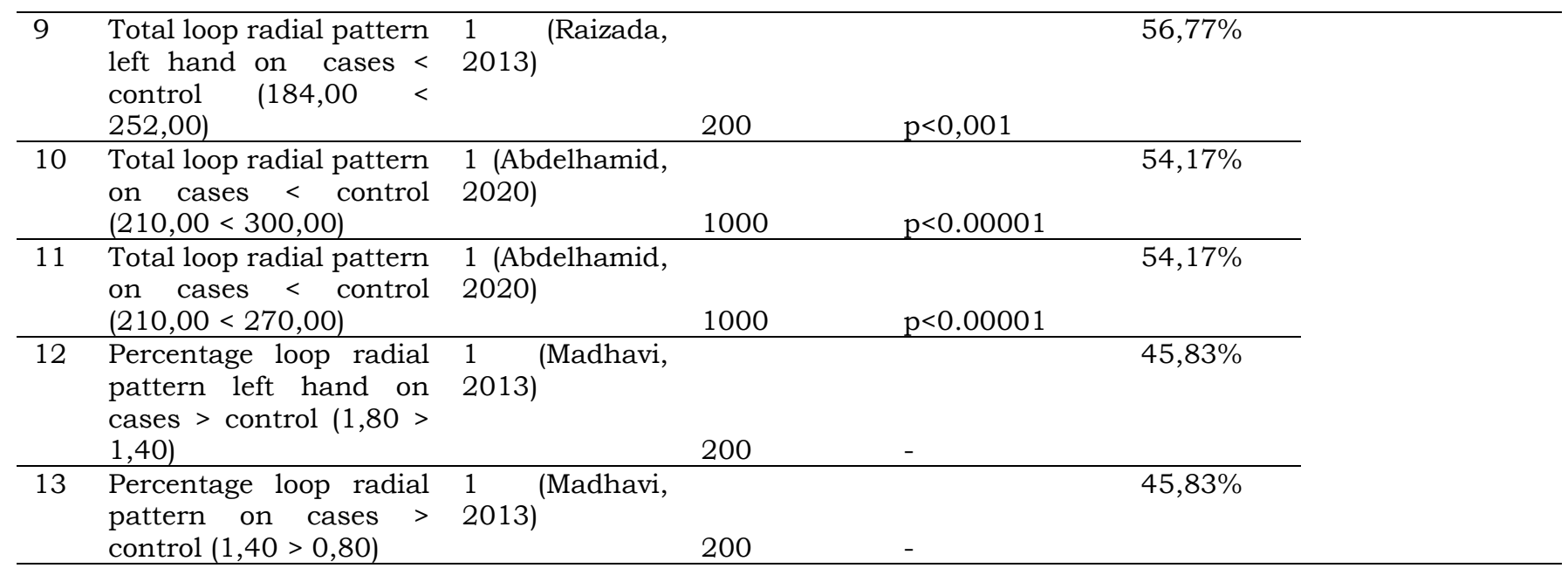

\section{Table 3. Breast cancer and loop ulnar pattern}

\begin{tabular}{|c|c|c|c|c|c|c|}
\hline \multicolumn{7}{|c|}{$\begin{array}{l}\text { Subgroup criteria } \\
\text { P: female, aged } 25-60 \text { years old } \\
\text { I: breast cancer based on histopathological evaluation } \\
\text { C: non- breast cancer } \\
\text { O: dermatoglyphy }\end{array}$} \\
\hline No & Results & $\begin{array}{l}\text { Number of } \\
\text { study }\end{array}$ & $\begin{array}{l}\text { Total } \\
\text { participants }\end{array}$ & $\begin{array}{ll}\text { P-value/ risk } \\
\text { ratio/ } & \text { odd } \\
\text { ratio/ } & \\
\text { confidence } & \\
\text { interval } & \end{array}$ & $\begin{array}{l}\text { Individual } \\
\text { bias risk }\end{array}$ & Notes \\
\hline 1 & $\begin{array}{l}\text { Percentage loop ulnar } \\
\text { pattern on cases < } \\
\text { control }(50,40<65,00)\end{array}$ & 1 (Fulari, 2012) & 100 & $\mathrm{p}<0,05$ & $51,04 \%$ & \multirow{12}{*}{$\begin{array}{l}\text { There are } \\
\text { studies which } \\
\text { state that there } \\
\text { are fewer ulnar } \\
\text { patterns in the } \\
\text { case group than } \\
\text { in the control } \\
\text { group with } \\
\text { value < } 0.05 \text { and } \\
\text { a good bias } \\
\text { assessment } \\
50 \% \text {. }\end{array}$} \\
\hline 2 & $\begin{array}{l}\text { Percentage loop ulnar } \\
\text { pattern on cases < } \\
\text { control }(49,70<64,40)\end{array}$ & $\begin{array}{l}1 \text { (Paranjape, } \\
2015)\end{array}$ & 200 & $\mathrm{p}<0,001$ & $52,60 \%$ & \\
\hline 3 & $\begin{array}{l}\text { Total loop ulnar pattern } \\
\text { on cases > control } \\
(493,00>481,00)\end{array}$ & $\begin{array}{l}1 \text { (Meghala, } \\
2020)\end{array}$ & 200 & $\mathrm{p}=0,0001$ & $42,71 \%$ & \\
\hline 4 & $\begin{array}{l}\text { Total loop ulnar pattern } \\
\text { on cases > control } \\
(985,00>222,00)\end{array}$ & 1 (Singh, 2020) & 290 & $\mathrm{p}<0,001$ & $48,96 \%$ & \\
\hline 5 & $\begin{array}{l}\text { Amount of loop ulnar } \\
\text { pattern }>6 \text { on cases }< \\
\text { control }(31,00<40,00)\end{array}$ & $\begin{array}{l}1 \text { (Musanovic, } \\
2019)\end{array}$ & 100 & $\mathrm{p}<0,05$ & $60,94 \%$ & \\
\hline 6 & $\begin{array}{l}\text { Amount of loop ulnar } \\
\text { pattern }<6 \text { on cases }< \\
\text { control }(19,00>10,00)\end{array}$ & $\begin{array}{l}1 \text { (Musanovic, } \\
2019)\end{array}$ & 100 & $\mathrm{p}<0,05$ & $60,94 \%$ & \\
\hline 7 & $\begin{array}{l}\text { Amount of loop ulnar on } \\
\text { cases }<\text { control }(32,00< \\
50,00)\end{array}$ & 1 (Sukre, 2012) & 100 & $\mathrm{p}<0,05$ & $47,92 \%$ & \\
\hline 8 & $\begin{array}{l}\text { Amount loop ulnar } \\
\text { pattern right hand on } \\
\text { cases }>\text { control }(25,00> \\
1,00)\end{array}$ & $\begin{array}{l}1 \\
2013)\end{array}$ (Raizada, & 200 & $\mathrm{p}<0,001$ & $56,77 \%$ & \\
\hline 9 & $\begin{array}{l}\text { Total loop ulnar pattern } \\
\text { left hand on cases > } \\
\text { control }(16,00>13,00)\end{array}$ & $\begin{array}{l}1 \\
2013)\end{array}$ (Raizada, & 200 & $P=0,-5$ & $56,77 \%$ & \\
\hline 10 & $\begin{array}{l}\text { Total loop ulnar pattern } \\
\text { right hand on cases }> \\
\text { control }(30,00>20,00)\end{array}$ & $\begin{array}{l}1 \text { (Abdelhamid, } \\
2020)\end{array}$ & 1000 & $\mathrm{p}<0.00001$ & $54,17 \%$ & \\
\hline 11 & $\begin{array}{l}\text { Total loop ulnar left } \\
\text { hand on cases }=\text { control } \\
(20,00=20,00)\end{array}$ & $\begin{array}{l}1 \text { (Abdelhamid, } \\
2020)\end{array}$ & 1000 & $\mathrm{p}<0.00001$ & $54,17 \%$ & \\
\hline 12 & Percentage loop ulnar & (Madhavi, & 200 & - & $45,83 \%$ & \\
\hline
\end{tabular}




\begin{tabular}{lllll}
\hline & pattern on cases $<2013)$ & & \\
& control $(34,40<76,80)$ & & $45,83 \%$ \\
\hline 13 & $\begin{array}{l}\text { Percentage loop ulnar } 1 \text { (Madhavi, } \\
\text { pattern on cases }<2013)\end{array}$ & \\
control $(34,60<77,00)$ & 200 & - & \\
\hline
\end{tabular}

Table 4. Breast cancer and arch pattern

\begin{tabular}{|c|c|c|c|c|c|c|}
\hline \multicolumn{7}{|c|}{$\begin{array}{l}\text { Subgroup criteria } \\
\text { P: female, aged } 25-60 \text { years old } \\
\text { I: breast cancer based on histopathological evaluation } \\
\text { C: non- breast cancer } \\
\text { O: dermatoglyphy }\end{array}$} \\
\hline No & Results & $\begin{array}{l}\text { Number of } \\
\text { studies }\end{array}$ & $\begin{array}{l}\text { Total } \\
\text { participant }\end{array}$ & $\begin{array}{ll}\text { P-value/ risk } \\
\text { ratio/ } & \text { odd } \\
\text { ratio/ } & \\
\text { confidence } & \\
\text { interval } & \\
\end{array}$ & $\begin{array}{l}\text { Individual } \\
\text { bias risk }\end{array}$ & Notes \\
\hline 1 & $\begin{array}{l}\text { Percentage arch pattern } \\
\text { on cases }<\text { control }(3,80 \\
<6,40)\end{array}$ & 1 (Fulari, 2012) & 100 & $\mathrm{p}>0,05$ & $51,04 \%$ & \multirow{16}{*}{$\begin{array}{l}\text { There are } 4 \\
\text { studies which } \\
\text { state that there } \\
\text { are fewer arch } \\
\text { patterns in the } \\
\text { case group than } \\
\text { the control group } \\
\text { with p value } \\
0.05 \text { and a good } \\
\text { bias assessment } \\
>50 \% \text {. }\end{array}$} \\
\hline 2 & $\begin{array}{l}\text { Percentage arch pattern } \\
\text { on cases }>\text { control }(7,40 \\
>3,50)\end{array}$ & $\begin{array}{l}1 \text { (Paranjape, } \\
2015)\end{array}$ & 200 & $\mathrm{p}<0,001$ & $52,60 \%$ & \\
\hline 3 & $\begin{array}{l}\text { Percentage arch pattern } \\
\text { on cases }>\text { control } \\
(38,00>24,00)\end{array}$ & $\begin{array}{l}1 \text { (Krishnan, } \\
2016)\end{array}$ & 200 & $\mathrm{p}=0,032$ & $45,83 \%$ & \\
\hline 4 & $\begin{array}{l}\text { Total arch pattern on } \\
\text { cases }<\text { control }(33,00< \\
79,00)\end{array}$ & $\begin{array}{l}1 \\
2016)\end{array}$ & 200 & $\mathrm{p}<0,05$ & $60,42 \%$ & \\
\hline 5 & $\begin{array}{l}\text { Total arch pattern on } \\
\text { cases }<\text { control }(16,00< \\
25,00)\end{array}$ & $\begin{array}{l}1 \text { (Meghala, } \\
2020)\end{array}$ & 200 & $p=0,0001$ & $42,71 \%$ & \\
\hline 6 & $\begin{array}{l}\text { Total arch pattern on } \\
\text { cases }>\text { control }(60,00> \\
255,00)\end{array}$ & 1 (Singh, 2020) & 290 & $\mathrm{p}<0,001$ & $48,96 \%$ & \\
\hline 7 & $\begin{array}{l}\text { Total arch pattern on } \\
\text { cases }>\text { control }(15,00> \\
10,00)\end{array}$ & $\begin{array}{l}1 \text { (Musanovic, } \\
2019)\end{array}$ & 100 & $p>0,05$ & $60,94 \%$ & \\
\hline 8 & $\begin{array}{l}\text { Total mean value arch } \\
\text { pattern on cases > } \\
\text { control }(33,00>29,00)\end{array}$ & $\begin{array}{l}1 \text { (Musanovic, } \\
2018)\end{array}$ & 232 & $\mathrm{P}=0,325$ & $60,94 \%$ & \\
\hline 9 & $\begin{array}{l}\text { Total arch pattern on } \\
\text { cases }>\text { control }(15,80> \\
8,40)\end{array}$ & 1 (Sukre, 2012) & 100 & $\mathrm{p}<0,05$ & $47,92 \%$ & \\
\hline 10 & $\begin{array}{l}\text { Total arch pattern right } \\
\text { hand on cases }>\text { control } \\
(180,00>57,00)\end{array}$ & $\begin{array}{l}1 \\
2013)\end{array}$ & 200 & $\mathrm{p}<0,001$ & $56,77 \%$ & \\
\hline 11 & $\begin{array}{l}\text { Total arch pattern left } \\
\text { hand on cases }>\text { control } \\
(199,00>81,00)\end{array}$ & $\begin{array}{l}1 \\
2013)\end{array}$ & 200 & $\mathrm{p}<0,001$ & $56,77 \%$ & \\
\hline 12 & $\begin{array}{l}\text { Total arch pattern on } \\
\text { cases }<\text { control }(32,00< \\
68,00)\end{array}$ & $\begin{array}{l}1 \\
2010)\end{array}$ & 200 & $\mathrm{p}<0,001$ & $55,73 \%$ & \\
\hline 13 & $\begin{array}{l}\text { Percentage arch pattern } \\
\text { on cases }<\text { control }(7,70 \\
<9,90)\end{array}$ & $\begin{array}{l}1 \text { (Shrivastava, } \\
2019)\end{array}$ & 218 & $p=0,069$ & $43,23 \%$ & \\
\hline 14 & $\begin{array}{l}\text { Total arch pattern right } \\
\text { hand on cases }<\text { control } \\
(20,00<40,00)\end{array}$ & $\begin{array}{l}1 \text { (Abdelhamid, } \\
2020)\end{array}$ & 1000 & $p<0.00001$ & $54,17 \%$ & \\
\hline 15 & $\begin{array}{l}\text { Total arch pattern left } \\
\text { hand on cases }<\text { control } \\
(20,00<70,00)\end{array}$ & $\begin{array}{l}1 \text { (Abdelhamid, } \\
2020)\end{array}$ & 1000 & $\mathrm{p}<0.00001$ & $54,17 \%$ & \\
\hline 16 & $\begin{array}{l}\text { Percentage arch pattern } \\
\text { on cases }>\text { control }\end{array}$ & $\begin{array}{l}1 \text { (Madhavi, } \\
\text { 2013) }\end{array}$ & 200 & - & $45,83 \%$ & \\
\hline
\end{tabular}




\begin{tabular}{lllll}
\hline & $(10,60>6,00)$ & & \\
\hline 17 & $\begin{array}{l}\text { Percentage arch pattern } \\
\text { on cases }>\text { control }(8,60\end{array}$ & 1 & 2013) \\
$>5,40)$ & & 200 & - & $45,83 \%$ \\
\hline
\end{tabular}

Table 5. Breast cancer and loops ulnar+radial pattern combination

\begin{tabular}{|c|c|c|c|c|c|c|}
\hline \multicolumn{7}{|c|}{$\begin{array}{l}\text { Subgroup criteria } \\
\text { P: female, aged } 25-60 \text { years old } \\
\text { I: breast cancer based on histopathological evaluation } \\
\text { C: non- breast cancer } \\
\text { O: dermatoglyphy }\end{array}$} \\
\hline No & Results & $\begin{array}{l}\text { Number } \\
\text { studies }\end{array}$ & $\begin{array}{l}\text { Total } \\
\text { participant }\end{array}$ & $\begin{array}{l}\mathrm{P} \text {-value/ risk } \\
\text { ratio/ odd ratio/ } \\
\text { confidence } \\
\text { interval }\end{array}$ & $\begin{array}{l}\text { Individual } \\
\text { bias risk }\end{array}$ & Notes \\
\hline 1 & $\begin{array}{l}\text { Percentage loops } \\
\text { ulnar+radial pattern on } \\
\text { cases }>\text { control }(98,00> \\
94,00)\end{array}$ & $\begin{array}{l}1 \text { (Krishnan, } \\
2016)\end{array}$ & 200 & $p>0,05$ & $45,83 \%$ & \multirow{6}{*}{$\begin{array}{l}\text { There is no } \\
\text { definitive } \\
\text { conclusion } \\
\text { regarding the } \\
\text { differences in the } \\
\text { ulnar+radial } \\
\text { loop pattern in } \\
\text { the case and } \\
\text { control groups. }\end{array}$} \\
\hline 2 & $\begin{array}{l}\text { Total loops ulnar+radial } \\
\text { pattern on cases }< \\
\text { control }(488,00< \\
540,00)\end{array}$ & $\begin{array}{ll}1 & \text { (Sakore, } \\
2016) & \end{array}$ & 200 & $\mathrm{p}<0,05$ & $60,42 \%$ & \\
\hline 3 & $\begin{array}{l}\text { Total loops ulnar+radial } \\
\text { pattern on cases > } \\
\text { control }(665,00> \\
592,00)\end{array}$ & $\begin{array}{ll}1 & \text { (Sridevi, } \\
2010) & \end{array}$ & 200 & $\mathrm{p}=0,011$ & $55,73 \%$ & \\
\hline 4 & $\begin{array}{ll}\text { Total pola } & \text { loops } \\
\text { ulnar+radial } & \text { pada } \\
\text { kasus }>\text { kontrol } & \\
\end{array}$ & & & & & \\
\hline 5 & $\begin{array}{l}\text { Percentage loops } \\
\text { ulnar+radial pattern on } \\
\text { cases < control }(47,43< \\
50,91)\end{array}$ & $\begin{array}{l}1 \text { (Shrivastava, } \\
2019)\end{array}$ & 218 & $\mathrm{p}=0,1$ & $43,23 \%$ & \\
\hline 6 & $\begin{array}{l}\text { Total loops ulnar+radial } \\
\text { pattern }<6 \text { on cases < } \\
\text { control }(31,00<38,00)\end{array}$ & $\begin{array}{l}1 \text { (Musanovic, } \\
2018)\end{array}$ & 232 & $\mathrm{P}=0,014$ & $60,94 \%$ & \\
\hline
\end{tabular}

Table 6. Breast cancer and TFRC

\begin{tabular}{|c|c|c|c|c|c|c|}
\hline \multicolumn{7}{|c|}{$\begin{array}{l}\text { Subgroup criteria } \\
\text { P: female, aged } 25-60 \text { years old } \\
\text { I: breast cancer based on histopathological evaluation } \\
\text { C: non- breast cancer } \\
\text { O: dermatoglyphy }\end{array}$} \\
\hline No & Results & $\begin{array}{l}\text { Number of } \\
\text { studies }\end{array}$ & $\begin{array}{l}\text { Total } \\
\text { participant }\end{array}$ & $\begin{array}{l}\text { P-value/ risk } \\
\text { ratio/ odd } \\
\text { ratio/ } \\
\text { confidence } \\
\text { interval }\end{array}$ & $\begin{array}{l}\text { Individual } \\
\text { bias risk }\end{array}$ & Notes \\
\hline 1 & $\begin{array}{l}\text { Total TFRC value on } \\
\text { cases }>\text { control } \\
(162,60>145,80)\end{array}$ & $\begin{array}{l}1 \\
2018)\end{array}$ & & $\mathrm{p}<0,05$ & $50,52 \%$ & \multirow{5}{*}{$\begin{array}{l}\text { There are } 3 \text { out of } \\
11 \text { studies where } \\
\text { the TFRC value in } \\
\text { cases is higher } \\
\text { than controls with } \\
\text { a p value }<0.05\end{array}$} \\
\hline 2 & $\begin{array}{l}\text { TFRC value }<70 \text { on } \\
\text { cases }>\text { control }(71,00> \\
15,00)\end{array}$ & $\begin{array}{l}1 \text { (Krishnan, } \\
2016)\end{array}$ & 200 & $\mathrm{p}=0,017$ & $45,83 \%$ & \\
\hline 3 & $\begin{array}{l}\text { TFRC value }>70 \text { on } \\
\text { cases }<\text { control }(29,00< \\
85,00)\end{array}$ & $\begin{array}{l}1 \text { (Krishnan, } \\
2016)\end{array}$ & 200 & $p>0,05$ & $45,83 \%$ & \\
\hline 4 & $\begin{array}{l}\text { TFRC value }>70 \text { on } \\
\text { cases }<\text { control }(45,26< \\
50,48)\end{array}$ & $\begin{array}{ll}1 & \text { (Sukre, } \\
2012) & \end{array}$ & 100 & $p>0,05$ & $47,92 \%$ & \\
\hline 5 & $\begin{array}{l}\text { Mean TFRC value on } \\
\text { cases > control }\end{array}$ & $\begin{array}{l}\text { 1, } \\
2010)\end{array}$ & 00 & $\mathrm{p}<0,001$ & $55,73 \%$ & \\
\hline
\end{tabular}






Table 7. Breast cancer and AFRC

\begin{tabular}{|c|c|c|c|c|c|c|}
\hline \multicolumn{7}{|c|}{$\begin{array}{l}\text { Subgroup criteria } \\
\text { P: female, aged } 25-60 \text { years old } \\
\text { I: breast cancer based on histopathological evaluation } \\
\text { C: non- breast cancer } \\
\text { O: dermatoglyphy }\end{array}$} \\
\hline No & Results & $\begin{array}{l}\text { Number of } \\
\text { studies }\end{array}$ & $\begin{array}{l}\text { Total } \\
\text { participant }\end{array}$ & $\begin{array}{l}\text { P-value/ risk } \\
\text { ratio/ odd ratio/ } \\
\text { confidence } \\
\text { interval }\end{array}$ & $\begin{array}{l}\text { Individual } \\
\text { bias risk }\end{array}$ & Notes \\
\hline 1 & $\begin{array}{l}\text { AFRC value }<80 \text { on } \\
\text { cases }>\text { control }(32,00> \\
18,00)\end{array}$ & $\begin{array}{l}1 \text { (Krishnan, } \\
2016)\end{array}$ & 200 & $\mathrm{p}=0,033$ & $45,83 \%$ & \multirow{2}{*}{$\begin{array}{l}\text { There is no } \\
\text { conclusion } \\
\text { regarding the } \\
\text { differences in } \\
\text { AFRC in the } \\
\text { case and control } \\
\text { groups }\end{array}$} \\
\hline 2 & $\begin{array}{l}\text { AFRC value }<80 \text { on } \\
\text { cases }<\text { control }(68,00< \\
82,00)\end{array}$ & $\begin{array}{l}1 \text { (Krishnan, } \\
2016)\end{array}$ & 200 & $\mathrm{P}>0,05$ & $45,83 \%$ & \\
\hline
\end{tabular}

Table 8. Breast cancer and a-b RC

\begin{tabular}{|c|c|c|c|c|c|c|}
\hline \multicolumn{7}{|c|}{$\begin{array}{l}\text { Subgroup criteria } \\
\text { P: female, aged } 25-60 \text { years old } \\
\text { I: breast cancer based on histopathological evaluation } \\
\text { C: non- breast cancer } \\
\text { O: dermatoglyphy }\end{array}$} \\
\hline No & Results & $\begin{array}{l}\text { Number of } \\
\text { studies }\end{array}$ & $\begin{array}{l}\text { Total } \\
\text { participant }\end{array}$ & $\begin{array}{l}\text { P-value/ risk } \\
\text { ratio/ odd ratio/ } \\
\text { confidence } \\
\text { interval }\end{array}$ & $\begin{array}{l}\text { Individual } \\
\text { bias risk }\end{array}$ & Notes \\
\hline 1 & $\begin{array}{l}\text { Mean value a-b RC on } \\
\text { cases }<\text { control } \\
(27,59<31,08) \text { right } \\
\text { hand }\end{array}$ & $\begin{array}{l}1 \\
\text { (Shrivastava, } \\
\text { 2019) }\end{array}$ & 218 & $\mathrm{p}=0.00000075$ & $43,23 \%$ & \\
\hline 2 & $\begin{array}{l}\text { Mean value a-b RC on } \\
\text { cases }<\text { control } \\
(28,50<32,87) \text { left } \\
\text { hand }\end{array}$ & $\begin{array}{l}1 \\
\text { (Shrivastava, } \\
\text { 2019) }\end{array}$ & 218 & $\mathrm{p}=0.0000001$ & $43,23 \%$ & \\
\hline 3 & $\begin{array}{l}\text { Mean value } \mathrm{a}-\mathrm{b} \mathrm{RC} \text { on } \\
\text { cases }<\text { control } \\
(73,80<81,82)\end{array}$ & $\begin{array}{l}1 \\
2012)\end{array}$ (Fulari, & 100 & $\mathrm{p}<0,05$ & $51,04 \%$ & $\begin{array}{l}\text { There is no } \\
\text { conclusion } \\
\text { regarding }\end{array}$ \\
\hline 4 & $\begin{array}{l}\text { a-b RC value }<30 \text { on } \\
\text { cases }>\text { control }\end{array}$ & $\begin{array}{l}1 \text { (Krishnan, } \\
2016)\end{array}$ & 200 & $p>0,05$ & $45,83 \%$ & $\begin{array}{l}\text { differences in a- } \\
\text { bridge count in }\end{array}$ \\
\hline
\end{tabular}




\begin{tabular}{|c|c|c|c|c|c|c|}
\hline & $(163,00>150,00)$ & & & & & the case and \\
\hline 5 & $\begin{array}{l}\text { a-b RC value }>30 \text { on } \\
\text { cases }<\text { control } \\
(37,00<50,00)\end{array}$ & $\begin{array}{l}1 \text { (Krishnan, } \\
2016)\end{array}$ & 200 & $p>0,05$ & $45,83 \%$ & control groups \\
\hline 6 & $\begin{array}{l}\text { Mean value a-b } \mathrm{RC} \text { on } \\
\text { cases }>\text { control } \\
(71,10>70,40)\end{array}$ & $\begin{array}{l}1 \text { (Yaneva, } \\
\text { 2018) }\end{array}$ & 142 & $\mathrm{p}<0.001$ & $50,52 \%$ & \\
\hline 7 & $\begin{array}{l}\text { Total a-b RC value on } \\
\text { cases }<\text { control } \\
(32,54<34,14)\end{array}$ & $\begin{array}{l}1 \\
2012)\end{array}$ (Sukre, & 100 & $\mathrm{p}<0,05$ & $47,92 \%$ & \\
\hline 8 & $\begin{array}{l}\text { Mean value a-b RC on } \\
\text { cases }<\text { control } \\
(31,75<37,80)\end{array}$ & $\begin{array}{l}1 \text { (Lavanya, } \\
2012)\end{array}$ & 60 & $\mathrm{p}<0,05$ & $33,33 \%$ & \\
\hline 9 & $\begin{array}{l}\text { Mean value a-b } \mathrm{RC} \text { on } \\
\text { cases }<\text { control } \\
(30,55<39,87)\end{array}$ & $\begin{array}{l}1 \text { (Madhavi, } \\
2013)\end{array}$ & 200 & $\mathrm{SE} 0,26$ & $45,83 \%$ & \\
\hline 10 & $\begin{array}{l}\text { Mean a-b RC value on } \\
\text { cases }<\text { control } \\
(30,52<39,66)\end{array}$ & $\begin{array}{l}1 \text { (Madhavi, } \\
2013)\end{array}$ & 200 & SE 0,22 & $45,83 \%$ & \\
\hline 11 & $\begin{array}{l}\text { Mean a-b } \mathrm{RC} \text { value on } \\
\text { cases }>\text { control } \\
(37,08>33,64)\end{array}$ & 1 (Gul, 2018) & 80 & $\mathrm{p}<0,001$ & $39,58 \%$ & \\
\hline 12 & $\begin{array}{l}\text { Mean a-b RC value on } \\
\text { cases }>\text { control } \\
(37,05>34,45)\end{array}$ & 1 (Gul, 2018) & 80 & $\mathrm{p}<0,001$ & $39,58 \%$ & \\
\hline
\end{tabular}

Table 9. Breast cancer and ATD angle

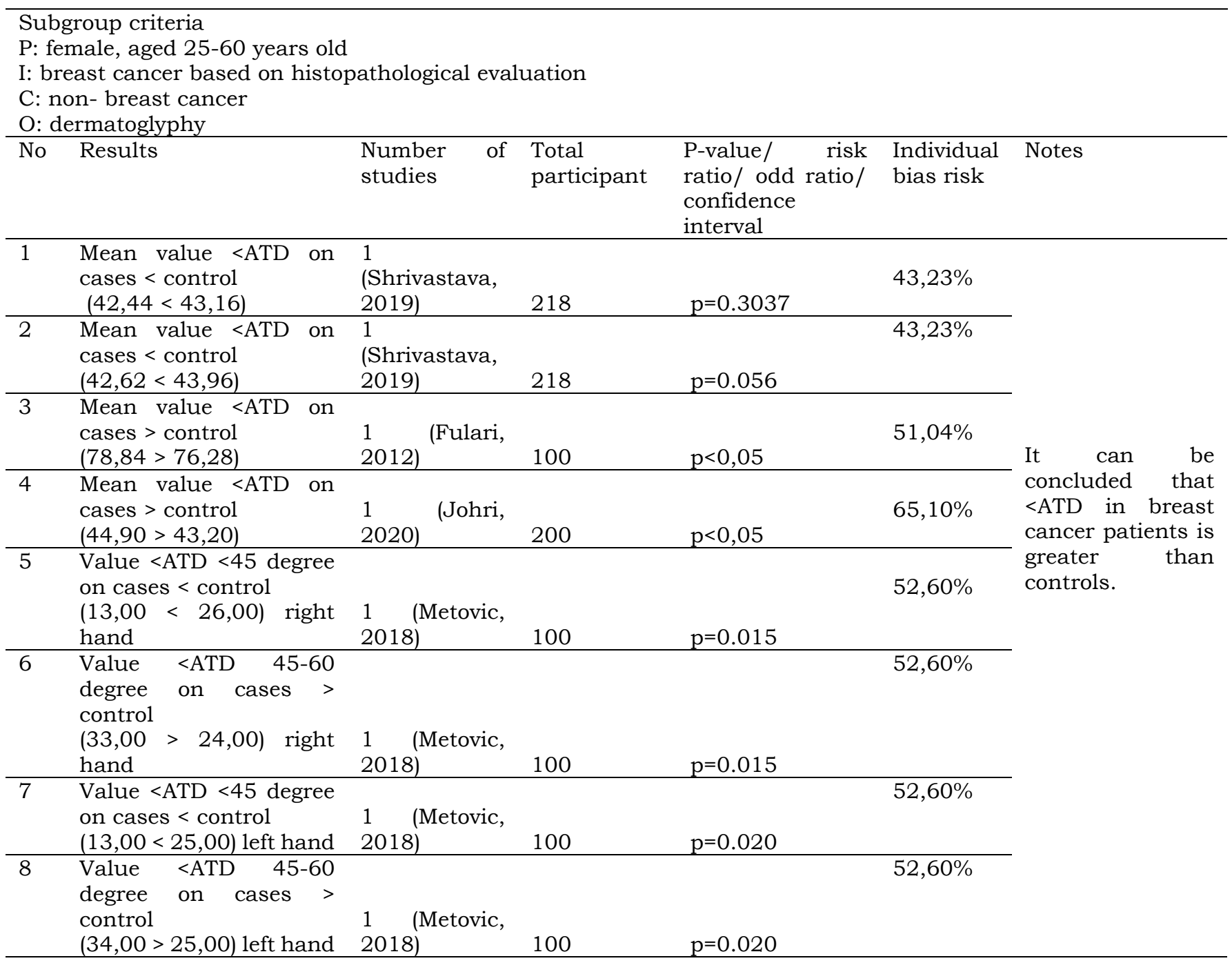




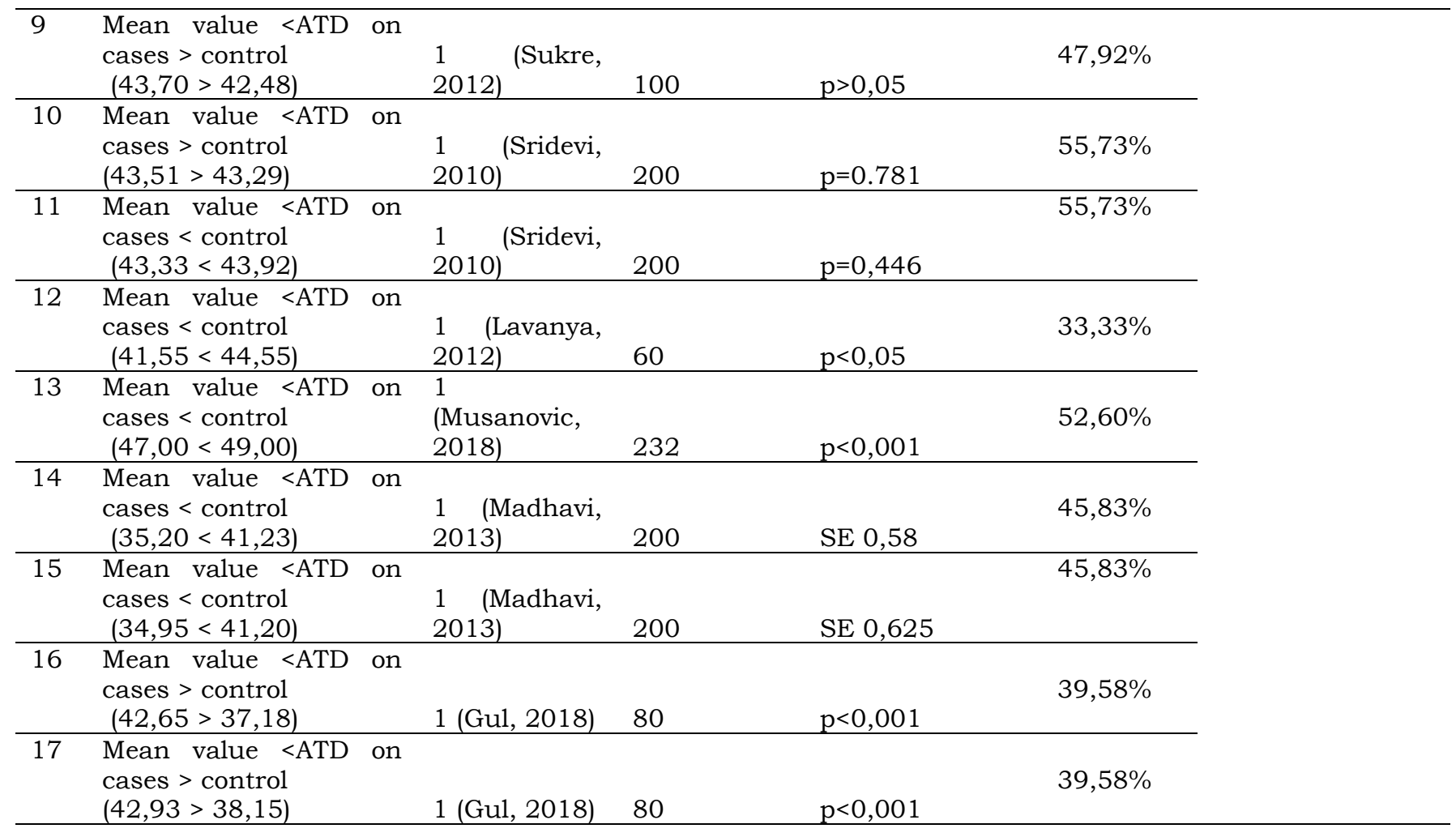

Table 10. Breast cancer and ADT angle

\begin{tabular}{|c|c|c|c|c|c|c|}
\hline \multicolumn{7}{|c|}{$\begin{array}{l}\text { Subgroup criteria } \\
\text { P: female, aged } 25-60 \text { years old } \\
\text { I: breast cancer based on histopathological evaluation } \\
\text { C: non- breast cancer } \\
\text { O: dermatoglyphy }\end{array}$} \\
\hline No & Results & $\begin{array}{l}\text { Number of } \\
\text { studies }\end{array}$ & $\begin{array}{l}\text { Total } \\
\text { participant }\end{array}$ & $\begin{array}{l}\text { P-value/ risk } \\
\text { ratio/ odd ratio/ } \\
\text { confidence } \\
\text { interval }\end{array}$ & $\begin{array}{l}\text { Individual } \\
\text { bias risk }\end{array}$ & Notes \\
\hline 1 & $\begin{array}{l}\text { Mean value <ADT on } \\
\text { cases }>\text { control } \\
(79,04>78,53) \text { on right } \\
\text { hand }\end{array}$ & $\begin{array}{l}1 \\
\text { (Shrivastava, } \\
\text { 2019) }\end{array}$ & 218 & $p=0.347$ & $43,23 \%$ & \multirow{6}{*}{$\begin{array}{l}\text { There is no } \\
\text { definitive } \\
\text { conclusion } \\
\text { regarding the } \\
\text { difference in } \\
\text { <ADT in the case } \\
\text { and groups. control } \\
\text { grous }\end{array}$} \\
\hline 2 & $\begin{array}{l}\text { Mean value <ADT on } \\
\text { cases }>\text { control } \\
(78,76>78,65) \text { on left } \\
\text { hand }\end{array}$ & $\begin{array}{l}1 \\
\text { (Shrivastava, } \\
\text { 2019) }\end{array}$ & 218 & $\mathrm{p}=0,87$ & $43,23 \%$ & \\
\hline 3 & $\begin{array}{l}\text { Mean value }<\text { ADT on } \\
\text { cases }<\text { control } \\
(76,40<77,70)\end{array}$ & $\begin{array}{l}1 \\
2020)\end{array}$ & 200 & $p>0,05$ & $65,10 \%$ & \\
\hline 4 & $\begin{array}{l}\text { Mean value <ADT on } \\
\text { cases }<\text { control } \\
(77,75<79,30) \text { on right } \\
\text { hand }\end{array}$ & $\begin{array}{l}1 \text { (Sridevi, } \\
2010)\end{array}$ & 200 & $p=0,028$ & $55,73 \%$ & \\
\hline 5 & $\begin{array}{l}\text { Mean value <ADT on } \\
\text { cases }<\text { control } \\
(77,61<79,41) \text { on left } \\
\text { hand }\end{array}$ & $\begin{array}{l}1 \text { (Sridevi, } \\
2010)\end{array}$ & 200 & $p=0,004$ & $55,73 \%$ & \\
\hline 6 & $\begin{array}{l}\text { Mean value <ADT on } \\
\text { cases }>\text { control } \\
(74,00>72,95) \text { right } \\
\text { hand }\end{array}$ & $\begin{array}{l}1 \text { (Madhavi, } \\
2013)\end{array}$ & 200 & SE 0,77 & $45,83 \%$ & \\
\hline
\end{tabular}




\begin{tabular}{lllll}
\hline 7 & Mean value <ADT on & & $45,83 \%$ \\
cases $>$ control & & & \\
$(73,70>72,70)$ left hand & $1 \quad$ (Madhavi, & & \\
& $2013)$ & 200 & SE 0,733 & \\
\hline
\end{tabular}

Table 11. Breast cancer and DAT angle

\begin{tabular}{|c|c|c|c|c|c|}
\hline \multicolumn{6}{|c|}{$\begin{array}{l}\text { Subgroup criteria } \\
\text { P: female, aged } 25-60 \text { years old } \\
\text { I: breast cancer based on histopathological evaluation } \\
\text { C: non- breast cancer } \\
\text { O: dermatoglyphy }\end{array}$} \\
\hline $\begin{array}{ll}\text { No } & \text { Results }\end{array}$ & $\begin{array}{l}\text { Number of } \\
\text { studies }\end{array}$ & $\begin{array}{l}\text { Total } \\
\text { participant }\end{array}$ & $\begin{array}{l}\text { P-value/ risk } \\
\text { ratio/ odd ratio/ } \\
\text { confidence } \\
\text { interval }\end{array}$ & $\begin{array}{l}\text { Individual } \\
\text { bias risk }\end{array}$ & Notes \\
\hline $\begin{array}{l}\text { Mean value }<\text { DAT on } \\
\text { cases }<\text { control } \\
(58,90<59,20)\end{array}$ & $\begin{array}{ll}1 & \text { (Johri, } \\
2020) & \end{array}$ & 200 & $\mathrm{p}>0,05$ & $65,10 \%$ & \multirow{3}{*}{$\begin{array}{l}\text { There is no } \\
\text { definitive } \\
\text { conclusion } \\
\text { regarding the } \\
\text { difference in } \\
\text { <DAT in the case } \\
\text { and groups } \\
\text { grontrol }\end{array}$} \\
\hline $\begin{array}{l}\text { Mean value <DAT on } \\
\text { cases }>\text { control } \\
(58,11>57,29)\end{array}$ & $\begin{array}{l}1 \text { (Sridevi, } \\
2010)\end{array}$ & 200 & $p=0,240$ & $55,73 \%$ & \\
\hline $\begin{array}{l}\text { Mean value }<\text { DAT on } \\
\text { cases }>\text { control } \\
(58,34>56,14)\end{array}$ & $\begin{array}{l}1 \text { (Sridevi, } \\
2010)\end{array}$ & 200 & $p=0,002$ & $55,73 \%$ & \\
\hline
\end{tabular}

Table 12. Breast cancer and triradial RC

\begin{tabular}{|c|c|c|c|c|c|}
\hline \multicolumn{6}{|c|}{$\begin{array}{l}\text { Subgroup criteria } \\
\text { P: female, aged } 25-60 \text { years old } \\
\text { I: breast cancer based on histopathological evaluation } \\
\text { C: non- breast cancer } \\
\text { O: dermatoglyphy }\end{array}$} \\
\hline No & Results & $\begin{array}{lll}\begin{array}{l}\text { Number } \\
\text { studies }\end{array} \text { of } & \begin{array}{l}\text { Total } \\
\text { participant }\end{array}\end{array}$ & $\begin{array}{l}\text { P-value/ risk } \\
\text { ratio/ odd ratio/ } \\
\text { confidence } \\
\text { interval }\end{array}$ & $\begin{array}{l}\text { Individual } \\
\text { bias risk }\end{array}$ & Notes \\
\hline 1 & $\begin{array}{l}\text { TRC mean value on } \\
\text { cases }>\text { control } \\
(18,90>16,74)\end{array}$ & $\begin{array}{l}1 \\
2012)\end{array}$ (Fulari, 100 & $\mathrm{P}<0,05$ & $51,04 \%$ & \multirow{2}{*}{$\begin{array}{l}\text { There is no } \\
\text { definitive } \\
\text { conclusion } \\
\text { regarding the } \\
\text { differences in } \\
\text { TRC in the case } \\
\text { and groups } \\
\text { grontrol }\end{array}$} \\
\hline 2 & $\begin{array}{l}\text { TRC mean value on } \\
\text { cases }<\text { control } \\
(11,59<11,89)\end{array}$ & $\begin{array}{l}1 \text { (Sukre, } 100 \\
2012)\end{array}$ & $p>0,05$ & $47,92 \%$ & \\
\hline
\end{tabular}

\section{Discussion}

Based on the review that has been done, there seems to be a relatively consistent finding of fingerprint patterns in breast cancer patients. For example, research by Fulari (2012), Paranjape (2015), Sakore (2016), and Abdelhamid (2020) reported that the whorl pattern was more often found in breast cancer patients than the control group with a p-value $<0.05$ and good bias assessment $>50 \%$. Meanwhile, several studies show the opposite, but the p-value is not significant, or the bias assessment is not good. Therefore, it can be concluded that breast cancer patients tend to have a whorl fingerprint pattern. This is supported by the Sakore research (2016), which states that there is a low Dankmeijer Index (DI) value while the Furuhata Index (FI) value increases accompanied by the results of Lavanya's research (2012), which found that a high Total Pattern Intensity (TPI) value where these three markers are closely related to a large number of whorl patterns.

Total pattern intensity (TPI) is the number of triradius found on all fingers, which is determined by counting the number of triradius on the ten fingertips per individual. The arch pattern is not counted because it does not have a triradius. The loop pattern is 
considered one because it has one triradius. The whorl pattern is considered two loops because it has two triradius. Meanwhile, DI is the division of the arch pattern with the whorl pattern multiplied by $100 \%$. The greater the number of whorls than the number of arches, the smaller the DI value and vice versa. FI is the division of the whorl pattern and the loop pattern multiplied by $100 \%$.

The more the number of whorls than the loop, the greater the FI value, and vice versa.

Furthermore, research by Paranjape (2015), Musanovic (2019), Raizada (2013), and Abdelhamid (2020) reported that the radial loop pattern was found to be less in the breast cancer group than the control group. The same thing was found in the ulnar loop fingerprint pattern in Fulari (2012), Paranjape (2015) and Musanovic (2019), and arch in Sakore (2016), Sridevi (2010) and Abdelhamid (2020) with p-value <0.05 and a good bias rating $>50 \%$. Meanwhile, there is no definitive conclusion regarding the differences in the combination of the ulnar+radial loop pattern in the case and control groups.

Another significant dermatoglyphic parameter found in the breast cancer group was the ATD angle. However, where the ATD angle in breast cancer patients was found to be more significant when compared to the control group in Fulari (2012), Johri (2015), and Metovic (2018) studies, for other parameters such as the value of TFRC, AFRC, a-bridge count, ADT angle, DAT angle, and Triradial RC there is no definitive conclusion that shows the difference between the two groups studied.

Several body structures developed simultaneously with the theoretical formation of dermatoglyphics. The body structures include the brain, breast glands, lips, and alveoli. Therefore, if a disorder occurs that causes developmental abnormalities of an organ that develops during the same period as dermatoglyphic formation, the abnormality of that organ will be reflected in the dermatoglyphic pattern that develops during gestation.[34]

There is an interaction of various genes that play a role in the control and development of dermatoglyphics in the fingers and palms; this can also indicate the development of premalignant and malignant diseases because breast development and fingerprint patterns develop at the same time during the intrauterine period. 5,35 However, in some cases, such as differences in ethnicity, race, certain geographic areas can also cause significant differences in genetic background. The frequency of fingerprint pattern types between one race, ethnicity, or nation can be different.13,25,28

\section{Conclusion}

Breast cancer patients tend to have more whorl fingerprint patterns and larger ATD angles, while for radial loop patterns, ulnar loops and arches are less in number when compared with the control group. Dermatoglyphics has the potential as an initial screening tool in at-risk groups. However, long-term studies and follow-up with larger sample sizes across ethnicities are needed to validate dermatoglyphics in anthropometric measurements as a promising marker of breast cancer.

\section{References}

1. Ferlay J, Colombet M, Soerjomataram I, Mathers C, Parkin DM, et al. Estimating the global cancer incidence and mortality in 2018 : GLOBOCAN sources and methods. Int $\mathrm{J}$ Cancer, 2019,144:1941-53.

2. Bray F, McCarron P, Parkin DM. The changing global patterns of female breast cancer incidence and mortality. Breast Cancer Res, 2004, 6:1-11.

3. Alkabban FM, Ferguson T. Breast Cancer. [Updated 2020 Nov 10]. In: StatPearls [Internet]. Treasure Island (FL): StatPearls Publishing; 2020 Jan-. Available from: https://www.ncbi.nlm.nih.gov/books/NBK48 2286/

4. Moore KL, Persaud TVN, Torchia MG. The developing human e-book: Clinically oriented embryology. 2018. Elsevier Health Sciences.

5. Bhat GM, Mukhdoomi MA, Shah BA, Ittoo MS. Dermatoglyphics: in health and disease-a review. Int J Res Med Sci, 2014, 2:31-7.

6. Jindal G, Pandey RK, Gupta S, Sandhu M. A 
comparative evaluation of dermatoglyphics in different classes of malocclusion. Saudi Dent J, 2015, 27:88-92.

7. Khandelwal R, Mittal A, Saijanani S, Tuteja A, Bansal A, et al. Qualitative and quantitative dermatoglyphic traits in patients with breast cancer: a prospective clinical study. BMC cancer, 2007, 7:1-5.

8. Nayak SB, Velan J, Shern NL, Zoung LF, Jeyarajan A, et al. Correlation between dermatoglyphic pattern of right thumb; learning methodologies; and academic performance of medical students. J Datta Meghe Inst Med Sci, 2017, 12:177.

9. Permatasari I, Wadji M, Adji RS. Hubungan sidik jari kaki dengan prestasi akademik pada mahasiswa Fakultas Kedokteran Universitas Diponegoro. Diponegoro Med J, 2019, 8:72334.

10. Pakhale, S.V., Borole, B.S., Doshi, M.A. and More, V.P., 2012. Study of the fingertip pattern as a tool for the identification of the dermatoglyphic trait in bronchial asthma. Journal of Clinical and Diagnostic Research: JCDR, 6(8), p.1397.

11. Varghese, S., 2016. Dermatoglyphics in congenital deafness. Journal of Evolution of Medical and Dental Sciences, 5(73), pp.53425348.

12. Mundijo, T. and Rezky, M., 2019. Dermatoglifi Narapidana di Palembang. Syifa'MEDIKA: Jurnal Kedokteran dan Kesehatan, 9(2), pp.8691.

13. Shrivastava, P. and Jain, A., 2019. Study of Palmar Patterns in Carcinoma Breast Patients of Central India. Int $\mathrm{J}$ Anat Res, 7(2.2), pp.6504-08.

14. Fulari, S.P., Pirzade, M.M. and Jadhav, A., 2012. Study of dermatoglyphics in breast cancer. National Journal of Medical Sciences, 1(1), pp.29-32.

15. Yaneva, G.A., Dimitrova, T.S., Cherneva, D.P., Ivanova, N.D., Fialkovska, N.A., Vachkov, I.H., Sivkov, S.T. and Ivanov, D.L., 2018.
Quantitative dermatoglyphic study of the finger ridge count in breast carcinoma patients from NortheastĐurn Bulgaria. Scripta Scientifica Salutis Publicae, 4, pp.51-55.

16. Paranjape, V., Kundalkar, A., Swamy, P.V. and Kulkarni, Y., 2015. Digital dermatoglyphics in carcinoma breast. International Journal of Current Research and Review, 7(21), p.47.

17. Johri, V., Raizada, A. and Takiar, R., 2020. Evaluation of Palmar Angles in Carcinoma Breast Patients and in Normal Healthy Females.

18. Krishnan, S. and Natesan, D., 2016. Dermatoglyphics in carcinoma breast. Journal of Evolution of Medical and Dental Sciences, 5(89), pp.6630-6634.

19. Yaneva, G., Dimitrova, T., Cherneva, D., Ivanova, N., Maslarski, I., Sivkov, S. and Ivanov, D., 2018. Comparative dermatoglyphic study of the palmar ridge count in breast carcinoma patients from Northeast Bulgaria. Acta morphologica et anthropologica, 25(1-2), pp.86-92.

20. Sakore Shubhangi, D., Waghmare Ramesh, S. and Kamble, R.A., 2016. Digital Dermatoglyphics in Carcinoma Breast. Indian Journal of Anatomy, 5(3), p.287.

21. Meghala, V. and Suganya, K., 2020. Digital dermatoglyphic study in patients on carcinoma breast. Journal of Natural Remedies, 21(4 (S1)), pp.44-50.

22. Singh, V., Jafar, S., Kaul, N. and Singh, B., 2019. Role of fingerprint patterns in the histopathologically diagnosed breast cancer females. Journal of the Anatomical Society of India, 68(3), p.211.

23. Musanovic, J., Metovic, A., Husremovic, F., Bejic, S., Haskovic, A., Babic, A., Sljuka, S. and Lepara, O., 2019. Qualitative analysis of digital dermatoglyphics in a small patients group in Bosnia and Herzegovina. Journal of Evolution of Medical and Dental Sciences, 8(17), pp.1415-1419.

24. Metovic, A., Musanovic, J., Alicelebic, S., Pepic, 
E., Sljuka, S. and Mulic, M., 2018. Predictive analysis of palmar dermatoglyphics in patients with breast cancer for small BosnianHerzegovinian population. Medical Archives, 72(5), p.357.

25. Sukre, S.B., Laeeque, M., Mahajan, A. and Shewale, S.N., 2012. Dermatoglyphics in the identification of women either with or at risk of breast cancer. Int J Basic Med Sci, 3(5), pp. 160-5.

26. Raizada, A., Johri, V., Ramnath, T., Chowdhary, D.S. and Garg, R.P., 2013. A cross-sectional study on the palmar dermatoglyphics in relation to carcinoma breast patients. Journal of clinical and diagnostic research: JCDR, 7(4), p.609.

27. Sridevi, N.S., Delphine Silvia, C.R., Kulkarni, R. and Seshagiri, C., 2010. Palmar dermatoglyphics in carcinoma breast of Indian women. Rom J Morphol Embryol, 51(3), pp.547-50.

28. Shrivastava, P. and Bose, A., 2019. Assessment of Digital Patterns in Carcinoma Breast Patients of Madhya Pradesh. Int J Anat Res, 7(1.2), pp.6225-29.

29. Lavanya, J., Saraswathi, P., Vijayakumar, J. and Prathap, S., 2012. Analysis of dermatoglyphic traits in patients with breast cancer. Journal of Pharmaceutical and Biomedical Sciences, 23(23).

30. Musanovic, J., Metovic, A., Pepic, E., Kapic, D., Cosovic, E., Rebic, D. and Lepara, O., 2018. Predictive values of quantitative analysis of finger and palmar dermatoglyphics in patients with breast cancer for Bosnian-Herzegovinian population. J Evolution Med Dent Sci, 7(24), pp.2855-2860.

31. Abdelhamid, M.I., Lotfy, M. and Awad, J.R., 2020. Fingerprint patterns, a novel risk factor for breast cancer in Egyptian populations: a case-kontrol study. The Egyptian Journal of Surgery, 39(1), p.247.

32. Madhavi, D., Dorairaj, S., Dorairaj, S.S.J. and Kommuru, H., 2016. Dermatoglyphic study in breast carcinoma patients. Int J Sci Res, 10, pp.8-6.

33. Shazya, G., Nusrat, J. and Sangeeta, G., 2018. Palmar dermatoglyphic and breast cancer: a possible correlation. International Journal of Medical and Health Research, 4(2), pp.53-5.

34. Suciandari, A. R., Mundijo, T., \& Purwoko, M. 2018. Dermatoglifi pada Autisme dan Sindrom Down di Palembang. Jurnal Berkala Ilmiah Kedokteran dan Kesehatan, 1(5).

35. Fuller IC. Inherited predisposition to cancer? A dermatoglyphic study. Br J Cancer, 1973, 28:186-9. 